\title{
Spinal health in 40 patients in the initial stage of laboural life. Morfogeometric, biological and environmental study.
}

\author{
José Vicente Martínez Quiñones', José Aso Escario', Cristina Sebastián Sebastián², Ignacio Bares Fernández², \\ Fabián Consolini Rossi', Ricardo Arregui Calvo ${ }^{1}$ \\ 1 Department of Neurosurgery and Neurology, Hospital MAZ, ${ }^{2}$ Department of Radiology, Hospital MAZ \\ Keywords: lumbar spine shape changes, Morphometrics, Genetics, spine \\ https://doi.org/10.52965/001c.30169
}

\section{Orthopedic Reviews}

Vol. 14, Issue 1, 2022

\section{Introduction}

Assuming that spinal shape is a genetic expression, its analysis and acquired factors could assess their respective contribution to early spine deterioration.

\section{Material and methods}

A geometric morphometric analysis was retrospectively performed on sagittal lumbar MRI of young patients with back pain to identify lumbar spine shape changes. Using Geometric Morphometrics, findings were analyzed with anthropometric, radiological, and clinical variables.

\section{Results}

80 cases under 26 years of age were collected, 55 men (mean age 22.81) and 25 women (mean age 23.24). MRI abnormalities were reported in 57.5\%: single altered disc $(\mathrm{N}=17)$, root compromises $(\mathrm{N}=8)$, and transition anomalies $(35 \%)$.

In the non-normal MRI subgroup, shape variation included: increased lordosis, enlarged vertebral body, canal stenosis, and lumbarization of S1. In non-Spanish origin patients, lumbar straightening and segmental deformities were prevalent. Morphometrics findings showed that lumbosacral transition anomalies are frequently underreported.

\section{Conclusions}

Genetic factors could be the main determinants of abnormality in MRIs under 26 years. The primary markers are transitional abnormalities, segmental deformities, and canal stenosis. In foreign populations, shape changes could suggest spine overload at an early age.

\section{INTRODUCTION}

Nowadays, it is considered a fact that most adults have suffered, or will suffer, from low back pain at some moment in their life. ${ }^{1}$

Recently, it has been published that chronic low back pain caused 60 million disabilities between 1990 and 2015 . The resulting disability was related to economic status, job satisfaction, financial compensation, and population aging. 2,3

Although low back pain is rare in the first decade of life, its prevalence increases during adolescence: $40 \%$ of people between 9 and 18 years of age, both in developed and undeveloped countries, report having suffered an episode of low back pain. ${ }^{4,5}$

Low back pain is closely related to the degeneration of the intervertebral disc, which would lead to a progressive deterioration of nearby structural elements, such as ligaments, joints, and muscles.

However, we do not know much about the causes and behavior of spinal disc pathology in young people. How is it possible for a person younger than 20 years to have a herniated disc if there has not yet been time for mechanical, traumatic, or nutritional factors to alter the disc components that start the degenerative cascade?

Should we consider, in these cases, the existence of a genetic predisposition that favors intervertebral disc degeneration?

Would any molecule originate after exposure to different environmental factors that silenced the expression of a gene that encodes a key enzyme to prevent the degradation of proteoglycans? ${ }^{6,7}$

When young people start working, they often suffer excessive or repeated mechanical requirements. In addition, young workers today come from different countries, some from the third world, in which health conditions and work 


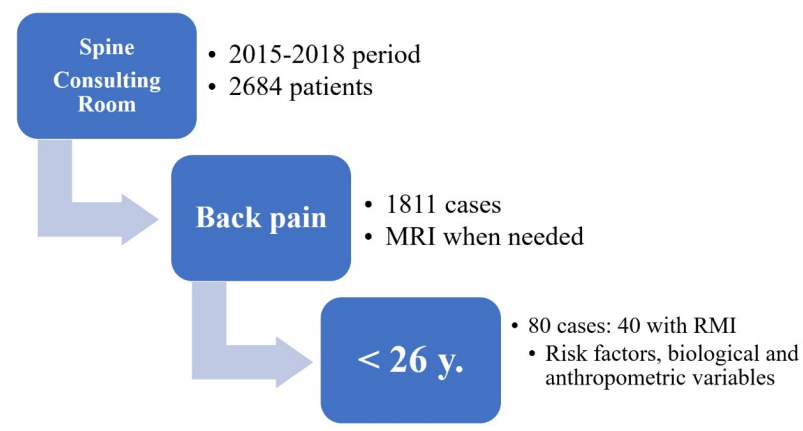

Figure 1.

Sample selection

environments are often more physically demanding than in developed countries.

All these possible etiologic factors could play a role in young disc degeneration, but their respective participation in low back pain remains undetermined.

According to Spanish legislation, the present work is based on patients under 26 years of age, with acute low back pain derived from an occupational accident (Royal Decree-Law 8/2015 of October 30, and Royal Decree-Law 28/ 2018 of December 28).

Our objective is to characterize this subpopulation clinically, radiologically, and morphologically, to investigate their spinal health and detect the principal or predisposing elements that may cause an early process of degeneration.

As a genetic-determined element, we analyze whether the spinal form constitutes a factor to consider in the origin of the disc-vertebral disease of the young working population.

\section{MATERIAL AND METHODS}

This is a retrospective study of patients under 26 years of age. They were collected from a specific spine consultation, created in January 2015, for patients referred to the Emergency Department suffering from spinal pain of occupational origin (occupational accident, according to the Spanish Law).

In the first 4 years, 2684 patients were seen in this consultation, 1811 of which were evaluated for low back pain. Of these, 80 patients were less than 26 years old.

The following anthropometric data were collected in this group: weight, height, abdominal circumference, body mass index (BMI), abdomen/height index (IAT). Other variables were sex, place of birth (Spanish or foreign), and other possible risk factors to which they could have been exposed (medical history, jobs performed, etc.). The patient selection methodology is summarized in Figure 1.

Due to an inadequate clinical course (low back pain refractory to treatment or persistent for more than 7 days), an MRI was performed in 40 patients. The equipment used was a Philips Ingenia 1.5T. We included sagittal sequences enhanced in SE T1, SE T2, STIR, and axial GE T2.
Evaluation of MRI images was performed by two radiologists, following standardized procedures: level of the most degenerated disc or with the probably symptomatic herniated disc; the number of discs with signs of intradiscal dehydration (minimum Pfirrmann grade II); mean disc height with a greater degree of disc degeneration and Pfirrmann grade of disc degeneration; presence or absence of annular fissure (in the STIR sequence) or disc herniation (protrusion, extrusion with or without migration, diffuse disc displacement); with a description of the type of hernia (focal, wide base) with or without root involvement. Cases with more than one herniated disc were collected. The existence of osteophytes, Schmörl's herniation, Modic changes, or facet arthropathy was also evaluated. Finally, the presence of other vertebral anomalies (significant scoliosis, spondylolisthesis, lumbosacral transition anomaly, or axial spondyloarthropathy) was analyzed. ${ }^{8-10}$

In addition, a study of the shape of the lumbar spine was carried out, using geometric morphometrics ${ }^{11}$ according to the following methodology: placement of landmarks in a single mid-sagittal T2 MRI section of each of the 40 patients with RM. The landmarks were transformed with the Procrustes superposition method to obtain an average image (consensus). Multivariate main component statistical analysis (PCA) was carried out using PAST. ${ }^{12}$ This technique allows us to know which morphological components explain the shape variability between the cases. MorphoJ software ${ }^{13}$ was used to find and represent the components that better identified the changes in the spine's shape, according to the percentage of variance explained by each of them (Figure 2).

The changes in shape were statistically analyzed along with other variables such as age, sex, body mass index, abdomen/height index, days of sick leave, origin, normality or not of the MRI, etc., to know the possible influence of these parameters on spinal shape changes. For this purpose, discriminant function analysis (DFA) or canonical variable analysis (CVA) were used.

\section{RESULTS}

Between 2015 and 2018, 80 patients under 26 years of age were treated, 55 men, with a mean age of 22.81 years (18 $25)$, and 25 women, with a mean age of 23.24 (19-25).

Males had a mean weight of $81.29 \mathrm{~kg}$, a mean height of $176.45 \mathrm{~cm}$, and an abdominal circumference of $96.22 \mathrm{~cm}$. Females had a mean weight of $62.95 \mathrm{~kg}$, a mean height of $161.46 \mathrm{~cm}$, and an abdominal circumference of $86.09 \mathrm{~cm}$.

There were no significant differences in the analysis of anthropometric data between males and females.

An MRI was performed in 40 of the 80 patients, 26 men (65\%) and 14 women (35\%).

There were no significant differences in the analysis of anthropometric data. We do find a statistically significant difference in the mean period of off days: $9.25+1.76$ (females) vs. $11.68+3.51$ (Males) $(2,5$ days $)(p=0.0305)$.

Radiological alterations were seen in $57.5 \%$ of the MRI studies, 18 men and 5 women (Table 1). Disc abnormalities 


\section{Landmarks}

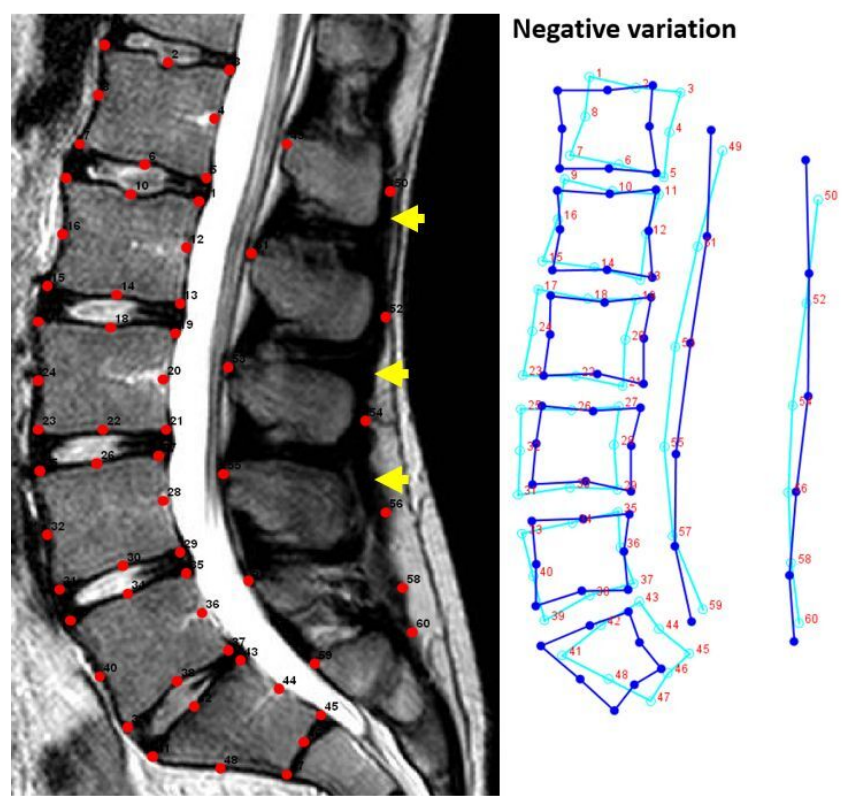

\section{Principal Components Analysis (PCA)}
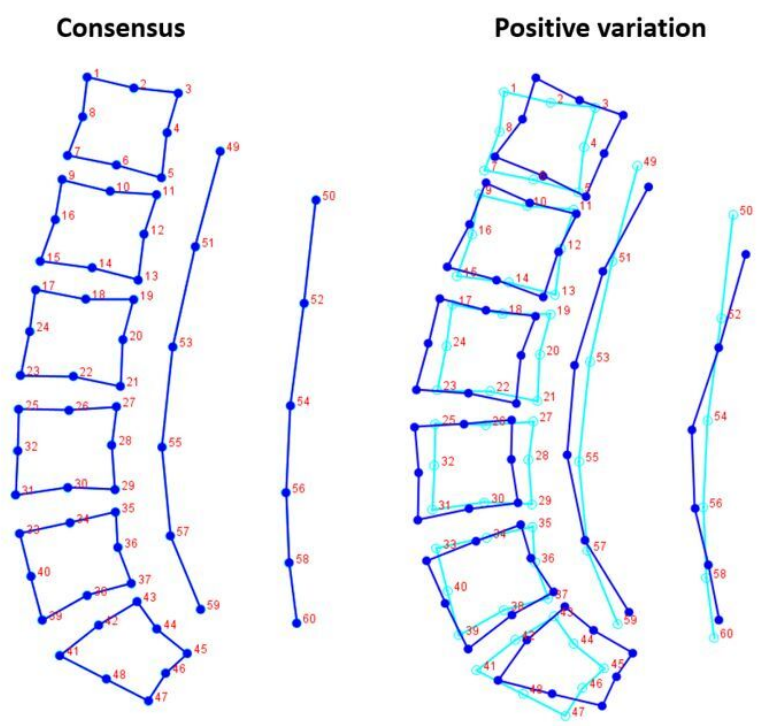

Figure 2.

Left: Digitization of landmarks (red dots). The yellow arrows represent the surface of the sacrolumbar common aponeurosis. The most posterior landmarks were placed on the lumbar fascia at the point of projection of the tip of the spinous process on it.

Right: Principal component analysis. Consensus and shape variations (in intense blue) of the lumbar sagittal balance, showing the shape changes corresponding to the main components.

Table 1. Summary of radiological abnormalities (MRI).

\begin{tabular}{|c|c|c|c|c|c|c|c|}
\hline \multicolumn{2}{|c|}{ Level } & \multicolumn{2}{|c|}{$\mathrm{N}^{\circ}$ of discs affected per patient } & \multicolumn{2}{|c|}{ Pfirrmann prevalent } & \multicolumn{2}{|c|}{ Morphological disc alteration } \\
\hline L1-L2 & 3 & 0 & 16 & I & 16 & Diffuse bulging & 4 \\
\hline L2-L3 & 1 & 1 & 17 & II & 11 & Protrusion & 19 \\
\hline L3-L4 & 4 & 2 & 3 & III & 9 & Extrusion & 4 \\
\hline L4-L5 & 4 & 3 & 4 & IV & 4 & & \\
\hline L5-S1 & 16 & 4 & 0 & & & & \\
\hline \multicolumn{2}{|c|}{ Osteophytes } & \multicolumn{2}{|c|}{ Modic changes } & \multicolumn{2}{|c|}{ Joint arthrosis } & \multicolumn{2}{|l|}{ Type of protrusion } \\
\hline YES & 3 & YES & 4 & YES & 6 & Wide basis. Posterocentral & 1 \\
\hline NO & 37 & NO & 36 & NO & 34 & Focal posterocentral & 11 \\
\hline & & & & & & Focal paracentral & 2 \\
\hline & & & & & & Focal foraminal & 5 \\
\hline \multicolumn{3}{|c|}{ Other significant alterations } & \multicolumn{3}{|c|}{ Other less significant alterations } & \multicolumn{2}{|l|}{ Root compression } \\
\hline \multicolumn{2}{|c|}{ Transition anomalies } & 14 & \multicolumn{2}{|c|}{ Espondilolisis } & 2 & YES & 8 \\
\hline \multicolumn{2}{|c|}{ Scoliosis } & 2 & \multicolumn{2}{|c|}{ Slight canal stenosis } & 2 & NO & 32 \\
\hline
\end{tabular}

were the most numerous finding, with a single disc affected in 17 patients and more than one in 7.

The most affected level was L5-S1, with 16 cases. The most prevalent morphological alteration was protrusion (19 cases), the most frequent feature of the posterocentral focal type (11 patients). Disc extrusions were observed in 4 patients. Root involvement was found in 8 patients. A finding to highlight is that lumbosacral transition anomalies were identified in $35 \%$ of the patients.
Of the 40 patients, 2 men needed surgical intervention (L5-S1 microdiscectomy). One of them required reoperation due to disc recurrence two years later.

The morphometric study has allowed us to appreciate that the variations by sex are the morphological changes that best explain the variability of the shape in this cohort. Through canonical variable analysis (CVA), statistically significant differences were identified between the MRs reported as non-normal compared to those reported as normal. 

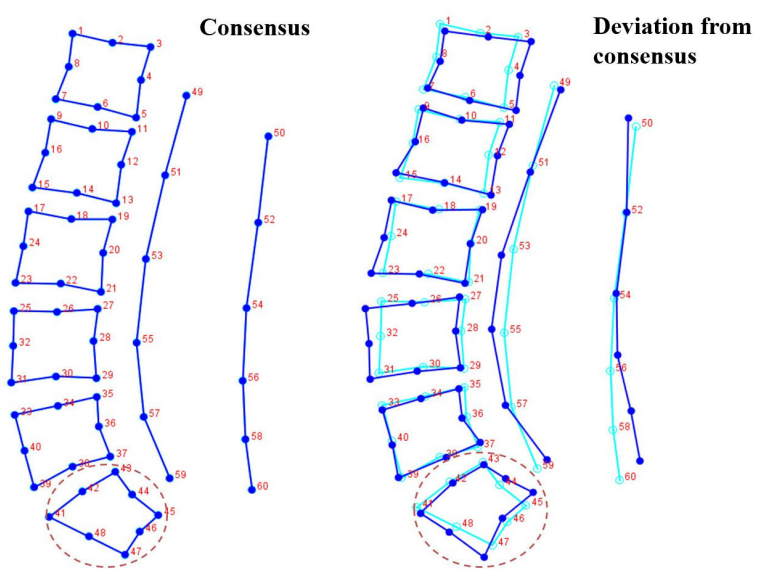

Figure 3.

Variations of shape corresponding to Canonical variable analysis of MRI. Main changes that allow discriminating normal from non-normal MRI are depicted. Note variations of caudal vertebral body (dotted circle) indicating strong differences representing transition anomalies in this region.

The changes in the abnormal MRI group were as follows: 1. Increased lordosis; 2 . Caudal deformation in lordosis of the tip spinous line (lumbar aponeurosis) 3. Tendency to vertebral body enlargement; 4 . Tendency to canal stenosis, and 5. Shape changes at the L5-S1 level, suggesting transitional anomalies (Figure 3).

Regarding the transition anomalies, it is noteworthy that the morphometric analysis of the MRI warned us about a possible more significant number of lumbosacral transition anomalies than those reported in the inform of MR.

In morphometrics study, a prevalent deformation of the caudal vertebra towards a lumbar morphology suggested a probable more significant occurrence of transitional abnormalities instead of sacral shape.

In the initial radiological review of the MR, only in 2 cases was identified the existence of lumbosacral transition anomalies. However, after the morphometric analysis, the radiologist reviewed the plain radiographs of all the cases, finding that 14 patients (35\% of the total) had unreported lumbosacral transition abnormalities.

Significant differences in shape were observed between patients of Spanish vs. foreign origin. The shape of the Spanish population was closer to consensus. In contrast, there were significant differences in the foreign population concerning the shape average, specifically: greater sagittal balance rectification and a greater number of segmental deformities (Figure 4).

No statistically significant variations in shape were found depending on BMI, IAT, tall or age.

\section{DISCUSSION}

Every disease has a molecular background. Our genome determines our vulnerability as a species. It is said that when we come into the world, we are born with around 60 "de novo" mutations, absent in the previous generation, which
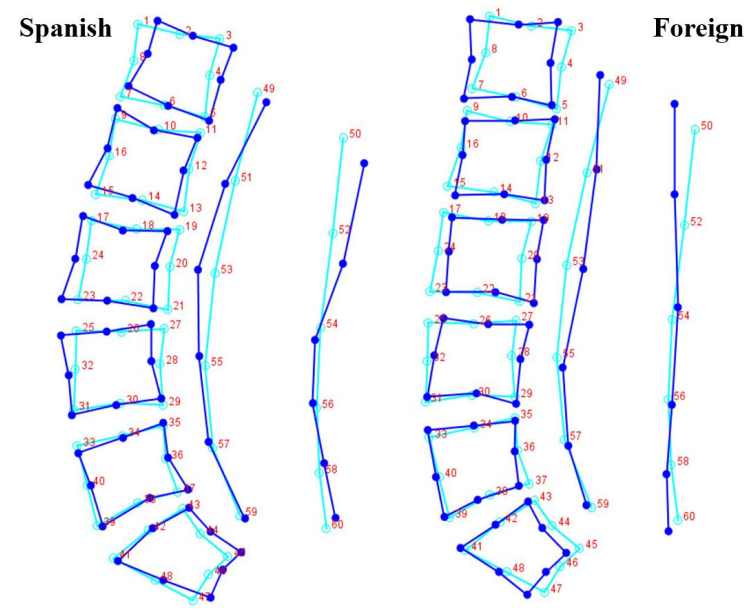

Figure 4.

Main shape changes representing the differences between the Spanish and foreign groups.

contributes to the genetic variation and evolution of our species. However, it sometimes causes diseases. ${ }^{14}$

Hundreds of genetic loci have been revealed to be associated with anthropometric peculiarities. Deep phenotyping is an emerging trend in precision medicine for congenital diseases. It is not only one trait at a time. Genetic variants affect body shape as a composite phenotype represented by anthropometric characteristics. ${ }^{15}$ For example, the shape of the face is involved in a variable percentage in many known genetic syndromes. ${ }^{16}$ Some gene mutations have been cited as a cause of spine deformity linked to degeneration. ${ }^{17}$

Our genome maintains a constant dialogue with the environment, in such a way that various external factors can cause epigenetic variations that could deactivate genes that should remain active, or activate others, out of place or at the wrong time, which should not, thus the development of diseases would be favored.

López Otín considers that, although we have "talents written in the genome," their expression will depend on the practice of the corresponding disciplines. In the same way, there would be genomic keys that would predispose to diseases. ${ }^{18}$

Recently Kawaguchi emphasized that two factors would favor lumbar disc disease, environmental factors (work activity, sports activity, smoking, etc.) and genetic factors. He argues that the era of epidemiological studies that used family history and the study of twins for the analysis of disc pathology is behind, to give way to genomic research using DNA polymorphism to identify which genes are likely to favor the development of disc disease. ${ }^{7}$

The work of Zitting et al., in a Finnish population, determined that the need for hospitalization due to lumbar disc herniation appears after the age of 15 , with the number of hospital admissions sharply increasing after the age of 19. 19

On the other hand, Jackson et al., reviewing the results of 40 publications on the prevalence of low back pain in 28 countries ( $n=80,076$ cases), found that chronic low back 
pain was 2.5 times more prevalent in the working population than in the non-working population, without clear causes to justify it. ${ }^{20}$ Spitzer et al. suggest that the onset of low back pain among the working class would occur between 20 and 24 years of age. ${ }^{21}$

Among the factors that can influence the appearance of low back pain in the first years of working life are a history of repeated trauma (most of them related to sports activities, falls, overexertion during adolescence, work, etc.), vertebral anomalies (scoliosis, transitional vertebrae, etc.) or genetic factors (between 13 and $57 \%$ of young adults with herniated discs have a first-degree relative with the same disorder). ${ }^{22}$

Considering that body shape is, in fact, an expression of the genome, an alternative way to the study of DNA polymorphism to assess the genetic origin of spine diseases could be the study of the morphology of the spine. Some works have used geometric morphometric methods to analyze different aspects of the spine. ${ }^{11,23}$

Our study demonstrates changes in the lumbar spine's shape, which correlate with the normality or abnormality reported in the MRI. These changes included: increased lordosis, increased vertebral sagittal enlargement, canal stenosis, and transition abnormalities.

Regarding lumbosacral transition anomalies, the incidence in our series is higher than that cited by the literature, which admits between $4-30 \%$ of the population. ${ }^{24}$ This could speak in favor of the fact that these anomalies are more prevalent in the subgroup of young and active people in symptomatic cases, which would have critical preventive consequences.

Furthermore, we observed a trend towards a higher degree of disc degeneration when a transitional anomaly coexisted. However, it did not reach statistical significance, probably due to the small number of patients.

These findings are in accordance with other studies that point to the possible influence of transitional abnormalities on disc degeneration in adjacent segments. ${ }^{25}$ Our work indicates that this association may also exist in young people, which would suggest that disc damage linked to these anomalies appears early. This has obvious preventive and occupational implications.

Lumbosacral transition abnormalities have a variable incidence $^{26}$ but cannot always be detected with the usual MRI protocols. ${ }^{27,28}$ We emphasize the need to perform complementary, simple spinal radiology studies for their adequate characterization ${ }^{28,29}$ to know its features and possible implications in disc alteration, particularly in young people.

These features point to the genetic component responsible for the shape change (transitional anomalies, increased anteroposterior vertebral diameter, and stenosis).
On the contrary, the influence on MRI normality or abnormality of BMI and abdominal circumference was not verified in this population group, despite their importance in older populations. ${ }^{11}$ At an early age, in which there is no degeneration, or it is incipient, it should be concluded that genetics is the main factor that promotes the shape variations observed in abnormal MRIs.

Some aspects of the environment can also explain the differences in the shape of the spine of foreign patients concerning native ones. More than racial factors, which would affect the entire spine, elements such as early overexposure to physical exertion or maintenance during childhood or adolescence, could explain the differences observed.

Consequently, our work points to the importance of genetic factors as the main determinants of abnormality of an MRI under 26 years of age. Transitional anomalies and canal and segmental deformities would be the primary markers.

In foreign patients, the presence of morphological alterations of the spine was greater than in autochthonous patients, which could also represent a marker of predisposition to lumbar problems.

Finally, as a protection factor, the group of females has a better prognosis in terms of healing days in this age range than the male. In studies on work-related low back pain, men have shorter sick periods than women. ${ }^{30,31}$

This study has a limitation: the size of the sample investigated, so it is challenging to obtain statically meaningful results. Despite this, our study is expected to provide clinically useful information to be considered and appropriately managed in the young subpopulation with back pain, both from a therapeutic and preventive point of view.

Likewise, further studies on genetic aspects of predisposition to low back pain due to vertebral pathology in the workplace are required.

\section{CONCLUSIONS}

Our study points to the importance of genetic factors and epigenetic variations as the most important factor in determining an anomaly in MRI under 26-year old's. The main indicators are lumbosacral transitional vertebrae, segment deformities, and spinal stenosis. Other acquired causes, as possible early spine overload, could also account for foreign patients.

We should take all these findings into account and use them appropriately both from a preventive and therapeutic point of view in back pain occurring in young people.

Submitted: September 23, 2021 EDT, Accepted: November 11, 2021 EDT 


\section{REFERENCES}

1. Lemeunier N, Leboeuf-Yde C, Gagey O. The natural course of low back pain: a systematic critical literature review. Chiropractic Man Ther. 2012;20(1):33-36. doi:10.1186/2045-709X-20-33

2. GBD 2015 DALYs and HALE Collaborators. Global, regional, and national disability-adjusted life-years (DALYs) for 315 diseases and injuries and healthy life expectancy (HALE), 1990-2015: a systematic analysis for the Global Burden of Disease Study 2015. Lancet. 2016;388(10053):1603-1658. doi:10.1016/S0140-673 $\underline{6(16) 31460-X}$

3. Urits I, Burshtein A, Sharma M, et al. Low Back Pain, a Comprehensive Review: Pathophysiology, Diagnosis, and Treatment. Curr Pain Headache Rep. 2019;23(3):23. doi:10.1007/s11916-019-0757-1

4. Calvo-Muñoz I, Gómez-Conesa A, Sánchez-Meca J. Prevalence of low back pain in children and adolescents: a meta-analysis. BMC Pediatr. 2013;13(1):1011861471-2431-13-14. doi:10.1186/147 1-2431-13-14

5. Louw QA, Morris LD, Grimmer-Somers K. The prevalence of low back pain in Africa: a systematic review. BMC Musculoskelet Disord. 2007;8(1):105-109. doi:10.1186/1471-2474-8-105

6. Sampara P, Banala RR, Vemuri SK, Reddy AVG, Subbaiah GPV. Understanding the molecular biology of intervertebral disc degeneration and potential gene therapy strategies for regeneration: a review. Gene Ther. 2018;25(2):67-82. doi:10.1038/s41434-01 8-0004-0

7. Kawaguchi Y. Genetic background of degenerative disc disease in the lumbar spine. Spine Surg Relat Res. 2018;2(2):98-112. doi:10.22603/ssrr.2017-0007

8. Buller M. MRI Degenerative Disease of the Lumbar Spine: A Review. J Am Osteopath Coll Radiol. 2018;7(4):11-19.

9. Pfirrmann CW, Metzdorf A, Zanetti M, Hodler J, Boos N. Magnetic resonance classification of lumbar intervertebral disc degeneration. Spine. 2001;26(17):1873-1878. doi:10.1097/00007632-20010 9010-00011

10. Modic MT, Herfkens RJ. Intervertebral disc: normal age-related changes in MR signal intensity. Radiology. 1990;177(2):332-334. doi:10.1148/radiolog y.177.2.2217764
11. Aso-Escario J, Sebastián C, Aso-Vizán A, Martínez-Quiñones JV, Consolini F, Arregui R. Body Mass Index and sagittal lumbar balance. A geometric morphometrics approach Eur J Anat. 2018;22(1):37-49.

12. Hammer $\emptyset$, Harper DAT, Ryan PD. PAST: Paleontological Statistics Software Package for Education and Data Analysis. Palaeontologia Electronica. 2001;4(1):9.

13. Klingenberg C. MorphoJ: an integrated software package for geometric morphometrics. Mol Ecol Resour. 2011;11(2):353-357. doi:10.1111/j.1755-099 8.2010.02924.X

14. Chakravorty S, Hegde M. Inferring the effect of genomic variation in the new era of genomics. Hum Mutat. 2018;39(6):756-773. doi:10.1002/humu.23427

15. Ried JS, Jeff MJ, Chu AY, et al. A principal component meta-analysis on multiple anthropometric traits identifies novel loci for body shape. Nat Commun. 2016;7(1):13357. doi:10.1038/nc omms 13357

16. Claes P, Roosenboom J, White JD, et al. Genomewide mapping of global-to-local genetic effects on human facial shape. Nat Genet. 2018;50(3):414-423. $\underline{\mathrm{d}}$ oi:10.1038/s41588-018-0057-4

17. Mäkitie RE, Niinimäki T, Suo-Palosaari M, et al. PLS3 Mutations Cause Severe Age and Sex-Related Spinal Pathology. Front Endocrinol (Lausanne). 2020;11:393. doi:10.3389/fendo.2020.00393

18. López Otín C. Los lenguajes de la vida. En: La vida en cuatro letras. Editorial Planeta; 2019:57-71.

19. Zitting $\mathrm{P}$, Rantakallio $\mathrm{P}$, Vanharanta $\mathrm{H}$.

Cumulative incidence of lumbar disc diseases leading to hospitalization up to the age of 28 years. Spine. 1998;23(21):2337-2344. doi:10.1097/00007632-19981 1010-00017

20. Jackson T, Thomas S, Stabile V, Shotwell M, Han $\mathrm{X}$, McQueen K. A Systematic review and metaanalysis of the global burden of chronic pain without clear etiology in low- and middle-income countries: trends in heterogeneous data and a proposal for new assessment methods. Anesth Analg. 2016;123(3):739-748. doi:10.1213/ane.000000000000 1389 
21. Spitzer WO, Leblanc FE, Dupuis M, et al. Scientific approach to the assessment and management of activity-related spinal disorders. A monograph for clinicians. Report of the Quebec Task Force on Spinal Disorders. Spine (Phila Pa 1976). 1987;12(7 Suppl):S1-59. doi:10.1097/00007632-198709001-0000 1

22. Dang L, Liu Z. A review of current treatment for lumbar disc herniation in children and adolescents. Eur Spine J. 2010;19(2):205-214. doi:10.1007/s0058 6-009-1202-7

23. Aso-Escario J, Aso-Vizán A, Martínez-Quiñones JV, Consolini F, Martín A, Arregui R. Dispositivos interespinosos y hernia discal. Estudio morfogeométrico y clínico de 71 casos tratados con microdiscectomía L4-L5 asociada a colocación de espaciador. Neurocirugía. 2015;26(4):180-191. doi:1 $\underline{0.1016 / \text { i.neucir.2014.11.007 }}$

24. Martín Noguerol T, Martínez Barbero JP, Broncano J. ¿L5 o S1? Valoración de las anomalías de transición lumbosacra mediante RM. European Society of Radiology (EPOS). Published online 2014. doi:10.15 94/seram2014/S-0844

25. Aihara T, Takahashi K, Ogasawara A, Itadera E, Ono Y, Moriya $\mathrm{H}$. Intervertebral disc degeneration associated with lumbosacral transitional vertebrae: a clinical and anatomical study. J Bone Joint Surg Br. 2005;87:687-691. doi:10.1302/0301-620X.87B5.15727
26. Taskaynatan MA, Izci Y, Ozgul A, Hazneci B, Dursun H, Kalyon TA. Clinical significance of congenital lumbosacral malformations in young male population with prolonged low back pain. Spine (Phila Pa 1976). 2005;30(8):E210-3. doi:10.1097/01.brs.0000 $\underline{158950.84470 .2 \mathrm{a}}$

27. Bressler EL. Numbering of lumbosacral transitional vertebrae on MRI. AJR Am J Roentgenol. 2007;188:W210, author reply W211. doi:10.2214/AI $\underline{\text { R.07.5201 }}$

28. Thawait GK, Chhabra A, Carrino JA. Spine Segmentation and Enumeration and Normal Variants. Radiol Clin North Am. 2012;50(4):587-598. doi:10.101 6/j.rcl.2012.04.003

29. Castellvi AE, Goldstein LA, Chan DP. Lumbosacral transitional vertebrae and their relationship with lumbar extradural defects. Spine. 1984;9(5):493-495. doi:10.1097/00007632-198407000-00014

30. Heymans MW, Anema JR, van Buuren S, Knol DL, van Mechelen W, de Vet HC. Return to work in a cohort of low back pain patients: development and validation of a clinical prediction rule. J Occup Rehabil. 2009;19(2):155-165. doi:10.1007/s10926-00 9-9166-3

31. Steenstra IA, Munhall C, Irvin E, et al. Systematic Review of Prognostic Factors for Return to Work in Workers with Sub Acute and Chronic Low Back Pain. J Occup Rehabil. 2017;27(3):369-381. doi:10.1007/s109 26-016-9666-x 